\title{
Science Beyond Calculations
}

Medical science is familiar with the Cartesian doctrine, in which proof and counterproof are fundamental to accept a specific factor observed. Moreover, almost always, it is advisable to present those proofs based on calculations that prove the results mathematically. Thus, the investigative actions are always based on rigid protocols in which, by specific methods, particularities of the phenomenon observed are measured and counted. Methods thus conceived are known as quantitative in scientific jargon. However, other investigative methods may be appropriate, especially in areas in which measuring and counting are almost impossible, or the observed phenomenon does not bear application of the quantitative method, or when this phenomenon is observed in another way during production of data.

In the present issue, the study of Pereira et al. is consistent with this other type of investigation. The method was not planned to measure or count variables, but to study these variables qualitatively. This type of study may cause some wonder, or even dismiss this type of reading among those used to read articles based on quantitative methods. However, their study has all the characteristic elements of a qualitative scientific investigation, producing results, discussion, and conclusion based on the results observed.

According to Black ${ }^{1}$, it is easier to accept results if they are expressed in numbers, i.e., quantified, even if the conclusion is reductionist or even unlikely. Qualitative investigators seek the truth beyond numbers. Their aim is to study the phenomenon in its natural context, trying to find its meaning, or interpret it, as a function of the meaning people attributes it. Thus, they use the "holistic perspective that preserves the complexities of human behavior" 2 . Nowadays, the thought that the quantitative approach excludes qualitative investigation has become obsolete. In the field of primary health care or investigation on health care, qualitative observation occupies a space increasingly more expressive. On many occasions, qualitative investigation rises as a prerequisite of quantitative investigation.

Quantitative investigation begins with an idea, which is transformed in hypothesis. This generates results and, by deduction, a conclusion. Qualitative investigation begins with the tendency to investigate a specific field. Data is collected by means of interviews, documents, and others. Through these data, ideas and hypothesis are created by inductive reasoning $^{2}$. Qualitative investigation cannot be repeated several times, always producing the same results. Its power is in validity, i.e., in how much it is close to the truth. It is not impervious and limited and it depends on the subjective experience of investigators and subjects ${ }^{3}$.

Physicians attribute a high value to results based in numbers, especially if they have many algorithms. We could give several examples of how many times we have been fooled by the same numbers when reality is different. The growing interest on qualitative investigations is due to the fact that quantitative methods often provide incorrect answers (or no answer) to clinical dilemmas and efficacy of health care.

In the past, medical journals rejected publication of studies with qualitative methodology, mostly due to their lack of knowledge of the investigative methodology used in these studies. Currently, they are opening space for qualitative investigations. Similar to quantitative investigation, it is also necessary to critically review the evidence presented.

Mário J Conceição, MD

Editor-in-chief

Revista Brasileira de Anestesiologia 


\section{REFERÊNCIAS / REFERENCES}

1. Black $\mathrm{N}$ - Why we need qualitative research. J Epidemiol Community Health, 1994;48:425-426.

2. Green J, Britten N- Qualitative research and evidence based medicine. BMJ, 1998;316:1230-1232.

3. Greenhalgh T - How to Read a Paper: the Basics of Evidence-Based Medicine. $3^{\text {rd }}$ Ed, Oxford, Blackwell Publishing, 2006;187-200. 